\title{
Cell proliferation of rat bladder urothelium induced by nicotine is suppressed by the NADPH oxidase inhibitor, apocynin
}

Suzuki Shugo, Cohen Samuel M., Arnold Lora L., Pennington Karen L., Gi Min, Kato Hiroyuki, Naiki Taku, Naiki-Ito Aya, Wanibuchi Hideki, Takahashi Satoru

\begin{tabular}{|c|c|}
\hline Citation & Toxicology Letters. 336; 32-38 \\
\hline Issue Date & 2021-01-01 \\
\hline Type & Journal Article \\
\hline Textversion & Author \\
\hline $\begin{array}{c}\text { Appendix. } \\
\text { Supplementary } \\
\text { materials }\end{array}$ & $\begin{array}{l}\text { Supplementary materials is available at } \\
\text { https://doi.org/10.1016/j.toxlet.2020.11.005 }\end{array}$ \\
\hline Rights & $\begin{array}{l}\text { (C) } 2020 \text { Elsevier B.V. This manuscript version is made available under the } \\
\text { CC-BY-NC-ND } 4.0 \text { License. http://creativecommons.org/licenses/by-nc-nd/4.0/. } \\
\text { This is the accepted manuscript version. The article has been published in final } \\
\text { form at https://doi.org/10.1016/j.toxlet.2020.11.005 }\end{array}$ \\
\hline DOI & 10.1016/j.toxlet.2020.11.005 \\
\hline
\end{tabular}

Self-Archiving by Author(s)

Placed on: Osaka City University Repository

Suzuki, S., Cohen, S. M., Arnold, L. L., Pennington, K. L., Gi, M., Kato, H., Naiki, T., Naiki-Ito, A., Wanibuchi, H., \& Takahashi, S. (2021). Cell proliferation of rat bladder urothelium induced by nicotine is suppressed by the NADPH oxidase inhibitor, apocynin. Toxicology Letters, 336, 32-38. https://doi.org/10.1016/j.toxlet.2020.11.005 
Cell Proliferation of Rat Bladder Urothelium Induced by Nicotine is Suppressed by the NADPH Oxidase Inhibitor, Apocynin

Shugo Suzuki ${ }^{1,2^{*}}$, Samuel M. Cohen ${ }^{3,4}$, Lora L. Arnold ${ }^{3}$, Karen L. Pennington ${ }^{5}$, Min Gi ${ }^{1}$

Hiroyuki Kato $^{2}$, Taku Naiki ${ }^{2}$, Aya Naiki-Ito ${ }^{2}$, Hideki Wanibuchi ${ }^{1}$ and Satoru Takahashi ${ }^{2}$

${ }^{1}$ Department of Molecular Pathology, Osaka City University Graduate School of

Medicine, Osaka, Osaka 545-8585, Japan

${ }^{2}$ Department of Experimental Pathology and Tumor Biology, Nagoya City University

Graduate School of Medical Sciences, Nagoya, Aichi 467-8601, Japan.

${ }^{3}$ Department of Pathology and Microbiology, University of Nebraska Medical Center,

Omaha, NE, 68198-5900, USA.

${ }^{4}$ Havlik-Wall Professor of Oncology, University of Nebraska Medical Center, Omaha,

NE, 68198-3135, USA

${ }^{5}$ Department of Pharmaceutical Sciences, University of Nebraska Medical Center,

Omaha, NE, 68198- 6849, USA

*To whom correspondence should be addressed.

TEL : +81-6-6645-3737

FAX : +81-6-6646-3093

Email : suzuki.shugo@med.osaka-cu.ac.jp

Keywords: Nicotine; urinary bladder carcinogenesis; oxidative stress; apocynin; cell proliferation. 


\begin{abstract}
Tobacco smoking is a major risk factor for human cancers including urinary bladder carcinoma. In a previous study, nicotine enhanced rat urinary bladder carcinogenesis in a two-stage carcinogenesis model. Nicotine also induced cytotoxicity in the bladder urothelium in a short-term study. In the present study, male rats were treated with nicotine $(40 \mathrm{ppm})$ in drinking water co-administered with the NADPH oxidase inhibitor, apocynin $(0,250$ or $750 \mathrm{mg} / \mathrm{kg})$ in diet for 4 weeks. The apocynin treatment induced no clinical toxic effects. Reduction of reactive oxygen species (ROS) by apocynin was confirmed by immunohistochemistry of $8-\mathrm{OHdG}$ in the bladder urothelium. Incidences of simple hyperplasia, cell proliferation and apoptosis were reduced by apocynin treatment in the bladder urothelium. However, despite reduction of cell proliferation (labeling index), apocynin did not affect the incidence of simple hyperplasia, apoptosis, or ROS generation in the kidney pelvis urothelium, in addition to $8-\mathrm{OHdG}$ positivity induced by nicotine being lower. In vitro, apocynin $(500 \mu \mathrm{M})$ reduced ROS generation, but induced cell proliferation in bladder cancer cell lines (T24 and UMUC3 cells). These data suggest that oxidative stress may play a role in the cell proliferation of the bladder urothelium induced by nicotine.
\end{abstract}




\section{Introduction}

Cigarette smoking is a major risk factor for numerous malignancies, including urothelial carcinoma of the urinary bladder, kidney pelvis and ureters (IARC, 2004). The risk of urinary bladder cancer in cigarette smokers is approximately 3 times higher than non-smokers (Baris et al., 2009). Although aromatic amines in cigarette smoke are considered the major cause of the urothelial malignancies in cigarette smokers by formation of DNA adducts and mutations (Cohen et al., 2006), the exposure levels do not fully explain the increased risk. Increased urothelial cell proliferation also occurs in cigarette smokers (Auerbach and Garfinkel, 1989) and in mice (Ohnishi et al., 2007) exposed to cigarette smoke, providing a synergistic interaction between the DNA reactive mutagenic activity of the aromatic amines and the proliferative effect. Nicotine is a major component of cigarette smoke and has been associated with increased cell proliferation in various carcinoma cell lines (Dasgupta et al., 2009; Minna, 2003). In previous studies, administration of nicotine in the drinking water to rats and to mice resulted in increased urothelial proliferation (Dodmane et al., 2014), and nicotine enhanced rat urinary bladder carcinogenesis induced by the DNA reactive N-butyl-N-(4-hydroxybutyl)nitrosamine (BBN) (Suzuki et al., 2018). We also showed that nicotinic acetylcholine receptors (nAChRs) were possibly involved with the inhibition of the urothelial cell proliferation and simple hyperplasia in rat urinary bladder and kidney pelvis induced by nicotine treatment (Suzuki et al., 2018). Interestingly, cotinine, a major metabolite of nicotine, also induced cell proliferation and simple hyperplasia of rat urinary bladder and kidney pelvis, but did not induce cytotoxicity detected by scanning electron microscopy (SEM) or apoptosis evaluated by the TUNEL assay or caspase immunohistochemistry in the bladder urothelium (Suzuki et al., 2020). Therefore, nicotine, not cotinine, may have a potential 
to induce cytotoxicity and regenerative proliferation in the bladder urothelium whereas cotinine is possibly acting as a direct mitogen.

A previous report indicated that nicotine induced genomic alterations which could be mediated through oxidative stress (Bavarva et al., 2014). Therefore, we focused on the relationship between nicotine and oxidative stress as a possible explanation at least in part for the effects of nicotine administration on the urinary bladder urothelium. Nicotinamide adenine dinucleotide phosphate (NADPH) oxidase is known to produce intracellular reactive oxygen species (ROS) and is implicated in a variety of signaling events, including cell growth, cell survival and cell death (Bedard and Krause, 2007). Apocynin, a methoxy-substituted catechol, inhibits NADPH oxidase by blocking the association of p47phox and p67phox with gp91phox (Stolk et al., 1994). We previously reported that apocynin reduced oxidative stress in arsenite-treated rat urothelium (Suzuki et al., 2009), and apocynin suppressed carcinogenesis of the pancreas and liver induced by various chemicals, and prostate in a transgenic model (Fuji et al., 2017; Kato et al., 2015; Suzuki et al., 2013b).

In the present study, we focused on the effects of apocynin on nicotine-induced cytotoxicity and consequent increased cell proliferation in a 4-week study of nicotine administration to rats, which is a useful model for studying the effects of nicotine on the urothelium of the urinary bladder and kidney pelvis (Dodmane et al., 2014; Suzuki et al., 2018; Suzuki et al., 2020).

\section{Materials and Methods}




\subsection{Chemicals}

Nicotine hydrogen tartrate (CAS No.: 65-31-6; purity $\geq 95 \%$ ) was purchased from Nacalai Tesque, Inc. (Kyoto, Japan). Apocynin (CAS No.: 498-02-2; purity $\geq 98 \%$ ) was purchased from Tokyo Chemical Industry Co., Ltd. (Tokyo, Japan). Cotinine (CAS No.: 486-56-6; purity $\geq 98 \%$ ) was purchased from Toronto Research Chemicals (North York, ON, Canada).

\subsection{Animals}

Animal experiments were performed under protocols approved by the Institutional Animal Care and Use Committee of Nagoya City University School of Medical Sciences. Five-week-old male F344 (F344/DuCrlCrlj) rats were obtained from Charles River Japan Inc. (Atusgi, Japan). They were housed in plastic cages with hardwood chip bedding in a room targeted at $23 \pm 2{ }^{\circ} \mathrm{C}$ and $55 \pm 5 \%$ humidity with a $12 \mathrm{hr}$ light/dark cycle. They were maintained on a basal diet (Oriental MF, Oriental Yeast Co., Tokyo, Japan) and tap water ad libitum.

\subsection{Animal experiment}

After 1 week in quarantine, the rats were randomly divided into 3 groups of 1213 animals each. Animals were co-administered 114 ppm nicotine hydrogen tartrate (40 ppm nicotine) in the drinking water and 0 (as positive control), 250 or $750 \mathrm{mg} / \mathrm{kg}$ apocynin in the diet for 4 weeks. The dose of apocynin was set based on the daily intake of apocynin in drinking water in a previous study (Fuji et al., 2017). A separate untreated control group was not included since spontaneous changes in the urothelium are rare (Cohen et al., 1990; 2007). Also, an apocynin only group was not included as it has been shown not to affect 
the rat urothelium when orally administered at these levels (Suzuki et al., 2009). The purpose of the present study was to see if apocynin had an effect on the nicotine-induced changes in the urothelium, so the nicotine only group served as the appropriate comparator. In a previous study, the serum cotinine concentration (approximately $350 \mathrm{ng} / \mathrm{ml}$ ) in rats treated with $114 \mathrm{ppm}$ nicotine hydrogen tartrate in the drinking water was similar to the serum cotinine concentration of active human smokers (250 to $300 \mathrm{ng} / \mathrm{ml}$ ) (Hukkanen et al., 2005; Suzuki et al., 2018). Nicotine supplemented drinking water solutions were prepared fresh weekly. During the experiment, body weights, food and drinking water consumption were measured weekly. At the end of week 4, under deep isoflurane anesthesia, the urinary bladder was inflated in situ with $10 \%$ buffered formalin, removed, and placed in $10 \%$ buffered formalin for 2 days. Rats then terminated by exsanguination while under deep isoflurane anesthesia. Liver and kidneys were removed, weighed and fixed in $10 \%$ buffered formalin. Following fixation, the bladders were rinsed in $70 \%$ ethanol and bisected longitudinally. One half of the bladder was processed for examination by SEM and classified in one of five categories as previously described (Cohen et al., 1990). Briefly, class 1 bladders have flat, polygonal superficial urothelial cells; class 2 bladders have occasional small foci of superficial urothelial cell death; class 3 bladders have numerous small foci of superficial urothelial cell death; class 4 bladders have extensive superficial urothelial cell death, especially in the dome of the bladder; and class 5 bladders have extensive cell death and piling up (hyperplasia) of rounded urothelial cells. Normal rodent urinary bladders are usually class 1 or 2 , or occasionally class 3 (Cohen et al., 1990; 2007). The other half of the bladder was cut longitudinally into strips, embedded in paraffin, stained with $H \& E$, and examined histopathologically and immunohistochemically. Urothelial hyperplasia represents an accumulation of cells and 
was diagnosed when the urothelium was greater than three cell layers thick. The liver and kidneys were processed routinely for histopathologic examination.

\subsection{Immunohistochemistry and TUNEL assays}

Deparaffinized sections of urinary bladder urothelium and kidney pelvis were immunohistochemically stained with either rabbit monoclonal anti-Ki67 (SP6, dilution 1:200, Abcam plc, Cambridge, UK) as a measure of DNA replication rate or anti-8hydroxy-2'-deoxyguanosine (8-OHdG) antibody (N45.1, dilution 1:100, Japan Institute for the Control of Aging, Fukuroi, Japan) as a measure of oxidative damage. Apoptotic cells in the urothelium and kidney pelvis were detected using an In Situ Apoptosis Detection Kit (TUNEL method) according to the manufacturer's instructions (Takara Bio Inc., Ohtsu, Japan). The number of Ki67, 8-OHdG or TUNEL-labeled cells in at least 1000 urothelial cells in each bladder and kidney were counted to determine labeling indices.

\subsection{Cell lines and conditions}

Human urinary bladder carcinoma cell lines, T24 (ATCC ${ }^{\circledR}$ HTB-4 ${ }^{\mathrm{TM}}$ ) and UMUC3 (ATCC ${ }^{\circledR}$ CRL-1749 ${ }^{\mathrm{TM}}$ ) were obtained from the American Type Culture Collection (ATCC; Manassas, VA, USA). The cell lines were tested for mycoplasma using the TaKaRa PCR Mycoplasma Detection Set (Takara Bio Inc., Kusatsu, Japan). All cell lines were routinely tested and authenticated using cell morphology, proliferation rate, a panel of genetic markers and/or contamination checks. All test materials were considered $100 \%$ pure for calculations of concentrations. T24 cells were grown in McCoy's 5A (modified) medium (Gibco-BRL, Grand Island, NY, USA) and 10\% sterile filtered FBS (Equitech-Bio, Inc., Kerrville, TX, USA). UMUC3 cells were cultured in E-MEM (Wako 
Pure Chemical Industries, Ltd., Osaka, Japan) and 10\% sterile filtered FBS. All cells were grown at $37^{\circ} \mathrm{C}$ in $5 \% \mathrm{CO}_{2}$.

\subsection{In vitro cell proliferation assay}

The cytotoxic and proliferative effects of apocynin and/or cotinine on T24 and UMUC3 cells were assessed by 4-[3-(4-iodophenyl)-2-(4-nitrophenyl)-2H-5-tetrazolio]1,3-benzene disulfonate tetrazolium salt (WST-1) assay (Roche Applied Science, Mannheim, Germany). Briefly, 1000 cells/well were seeded in 96-well plates in $100 \mu \mathrm{L}$ of culture medium. Apocynin and/or cotinine were added $24 \mathrm{hrs}$ after seeding and cells were incubated for three days. WST-1 reagent was added to each well, incubated for $3 \mathrm{hrs}$ at $37^{\circ} \mathrm{C}$, and then the absorbance of each well was measured at $450 \mathrm{~nm}$.

\subsection{In vitro detection of ROS production.}

$1 \times 10^{5}$ cells/well were seeded in 12 -well plates in $1 \mathrm{~mL}$ of culture medium. Apocynin were added 24 hrs after seeding, and cells were incubated for $24 \mathrm{hrs}$. After apocynin treatment, 2',7'-dichlorofluorescin-diacetate $(100 \mu \mathrm{g} / \mathrm{ml}$, DCFH-DA, SigmaAldrich, Co.) was added with further incubation at $37^{\circ} \mathrm{C}$ for $30 \mathrm{~min}$ in the dark. The cells were washed 2 times with warm PBS, and images were recorded with a fluorescence microscope (BZ-X710; Keyence Co., Osaka, Japan). For each sample, the average intensity of cells in five randomly selected views ( $\times 200$ magnification) was determined.

\subsection{Statistical analyses}

Statistical analyses were performed with mean \pm standard deviation (S.D.) using 1-way ANOVA and Dunnett's test or Tukey's multiple comparisons test by Prism ver. 6 
(GraphPad Software, Inc., La Jolla, CA). Differences in the incidences of histopathological lesions between control and apocynin-treated groups were evaluated by 2-tailed Fisher's exact test. Differences of $\mathrm{P}<0.05$ were considered statistically significant.

\section{Results}

\subsection{Body and Organ Weights, Water consumption, and histology of liver}

During the experimental period, minimal, stattistically not significant differences in body weight gain were observed among the groups, and final body, liver and kidneys weights did not significantly vary between groups (Table 1). There were no significant differences in water and food consumptions among the groups. There were no histolocial changes in liver tissue among the groups (data not shown).

\subsection{Histopathology and Ki67, TUNEL and 8-OHdG labeling indices, and SEM in the}

\section{bladder urothelium}

Histologically, simple hyperplasia was detected in the bladder urothelium of all groups of nicotine-treated rats (Fig. 1A, Table 2). Co-administration of apocynin with nicotine reduced the incidence of simple hyperplasia in a dose dependent manner, with a significant reduction at $750 \mathrm{mg} / \mathrm{kg}$ (Fig. 1A, Table 2). There was a significant decrease, in a dose-dependent manner, in the labeling indices of Ki67, TUNEL and 8-OHdG in the bladder urothelium of nicotine/apocynin-treated rats (Table 2). There was little difference by SEM classification among the groups based on the previously established scoring system (Cohen et al., 1990) (Supplementary Fig. 1, Table 2).

3.3. Histopathology and Ki67, TUNEL and 8-OHdG labeling indices in the kidney pelvis 
urothelium

In the kidney pelvis, simple hyperplasia was detected in the urothelium of all groups of nicotine-treated rats (Fig. 1B, Table 3). Co-admininstration of apocynin with nicotine did not reduce the incidence of simple hyperplasia induced by nicotine (Fig. 1B, Table 3). Apocynin co-administered with nicotine reduced the Ki67 labeling index in the urothelium of the kidney pelvis. The TUNEL and 8-OHdG labeling indices of the kidney pelvis urothelium were not altered by co-administration of apocynin with nicotine (Table 3). There were few changes in the glomeruli and kidney tubules, and were those typically seen with early chronic progressive nephropathy, a lesion commonly seen in rats (data not shown).

\subsection{Effect of cell growth in vitro study}

Apocynin significantly increased cell growth in vitro in the human urothelial carcinoma cell lines, T24 and UMUC3 (Fig. 2A). Co-administration of apocynin and cotinine induced significantly higher cell growth than that of either chemical alone (Fig. 2B). The effect was additive rather than synergistic. Apocynin significantly reduced ROS generation detected by DCFH-DA in both cell lines (Fig. 2C).

\section{Discussion}

The administration of nicotine alone produced simple hyperplasia of the urothelium with cytotoxicity detected by light microscopy and by SEM of the urinary bladder, similar to what was previously reported (Suzuki et al., 2018; 2020). Administration of oral nicotine has a possibility of metabolic inactivation in the liver during the first pass (Alsharari et al., 2014), but significant levels are excreted in the urine along 
with high concentrations of cotinine and other metabolites. Clearly, sufficient amounts of nicotine and its metabolites are excreted in the urine to produce an effect on the urothelium. In this study, we determined that co-administration of apocynin with nicotine reduced the incidence of simple hyperplasia, cell proliferation rate, apoptosis, and oxidative stress in the urothelium of the urinary bladder. Similar treatment for 4 weeks of apocynin previously was shown not to produce histological changes, cell proliferation, or oxidative stress on the urothelium of F344 rats (Suzuki et al., 2009). These data suggest that apocynin reduced oxidative stress in the urothelium, indicating that oxidative stress played a role in nicotineinduced urothelial effects. In contrast, the extent of surface cytotoxicity of the urothelium as detected by SEM was not affected, suggesting that apocynin effects are on the consequent cell proliferation. Apocynin is known to inhibit the assembly of NADPH oxidase that is responsible for ROS production (Stefanska and Pawliczak, 2008). Therefore, apocynin can only reduce intracellular ROS generation induced by NADPH oxidase. Other intracellular and/or extracellular ROS generation mechanisms may be induced by nicotine on the urothelium, but additional studies are needed to clarify the mechanism of nicotine-induced cytotoxicity, possibly using direct acting antioxidants.

Co-administration of apocynin with nicotine had no effect on the incidence of simple hyperplasia, apoptosis, or oxidative stress in the urothelium of the kidney pelvis. The cell proliferation rate in the urothelium of the kidney pelvis was significantly reduced by co-administration of apocynin with nicotine ( $30 \%$ reduction), but the decrease was less than that in the urothelium of the bladder (60\% reduction). Different effect in the kidney pelvis compared to the urinary bladder could be due to the short time the urine, with the chemcals, has in the kidney pelvis. Because the positivity of 8-OHdG in the urothelium of the kidney pelvis was approximately 40 times lower than that of the urinary bladder, the 
cytotoxic effects of nicotine in the urothelium of the kidney pelvis would be expected to be much lower than that of the urinary bladder. In a previous study, both nicotine and cotinine induced simple hyperplasia and cell proliferation, but did not induce apoptosis in the urothelium of the kidney pelvis (Suzuki et al., 2020). In addition, the data suggested that cotinine-induced cell proliferation in vitro was regulated via $\mathrm{nAchR}$, indicating that the effects of nicotine administration on the urothelium of the kidney pelvis may be due to a cytotoxic effect of nicotine itself (or some metabolite other than cotinine) and to a nAchR regulated pathway induced by cotinine (Beckel et al., 2006). This dual action might explain why apocynin had less of an effect in the kidney pelvis urothelium than in the urinary bladder, in addition to its shorter time in the kidney pelvis. Some ROS generation in the urothelium of both kidney pelvis and bladder may be related to the cell proliferation, which is reduced by apocynin, but may not be the main pathway in the kidney pelvis.

The cytotoxic effect of nicotine with consequent regenerative proliferation was present in the bladder urothelium in this study. Apocynin inhibited apotosis induced by nicotine with reduction of oxidative stress detected by $8-\mathrm{OHdG}$. Based on these results, the cytotoxic effects of nicotine in vivo may be due in part to oxidative stress in addition to a receptor-mediated process. This hypothesis is supported by the finding in the previous study that nicotine-induced cytotoxicity detected by SEM was decreased but not statistically significantly by nAChR inhibitors (Suzuki et al., 2018).

In the in vitro study, surprisingly, apocynin itself induced cell proliferation in both urothelical carcinoma cell lines. We also confirmed that apocynin reduced the ROS generation, as detected by DCFH-DA, in both cell lines via NADPH oxidases which were reported to be elevated in urinary bladder cancers (Miyata et al., 2017). In bladder cancer cell lines, some chemicals were reported to induce oxidative stress, apoptosis and inhibition 
of cell proliferation (Jiang et al., 2014; Qiu et al., 2015), but apocynin may have opposite effects. In the in vivo study, nicotine induced cytotoxicity and apoptosis in the bladder urothelium. The pathway for apoptosis induced by oxidative stress may be the same in both in vitro and in vivo studies. Meanwhile, apocynin is also reported to decrease levels of active Rac1 in some cancer cell lines (Stefanska and Pawliczak, 2008; Suzuki et al., 2013a). However, activation of Rac1 inhibited apoptosis in T24 cells (Pervaiz et al., 2001). The Rac1 pathway may not be regulated by apocynin in this study. Also, this proliferative effect of apocynin was in vitro and acting on malignant urothelial cells, which is in contrast to the lack of effect in vivo on normal urothelium (Suzuki et al., 2009).

In a previous study, we determined that cotinine induced cell proliferation via the nAchR in these cells (Suzuki et al., 2020). The effect of co-administration of apocynin with cotinine was additive rather than synergistic in both bladder cancer cell lines. These data suggest that the effect on cell proliferation may be via a nAchR regulated pathway and oxidative stress in the bladder urothelium. Nevertheless, the role of oxidative stress in the nicotine-induced effects on the urothelium in vivo appears to be complex and only partly explains the proliferative effects.

In summary, the present study suggests that oxidative stress may play a role in nicotine-induced cell proliferation as it was suppressed by the NADPH oxidase inhibitor, apocynin. Cell prolifeartion induced via mainly nAchR by nicotine in the urothelium of kidney pelvis was less effected by apocynin. Nicotine has the potential to induce urothelial cell proliferation via a complex interaction of various biologic effects including an effect on nAchR related pathway and induction of oxidative stress in the urinary bladder.

\section{Acknowledgements}


We gratefully acknowledge the expert technical assistance of Koji Kato and Junko Takekawa. The research was supported in part by funds from Nagoya City University and funds from the endowed professorship to Dr. Cohen at UNMC.

Conflict of Interest Statement: None declared. 


\section{References}

Auerbach, O., Garfinkel, L. 1989. Histologic changes in the urinary bladder in relation to cigarette smoking and use of articificial sweeteners, Cancer 64: 983-987.

Alsharari, S.D., Siu, E.C., Tyndale, R.F., Damaj, M.I., 2014. Pharmacokinetic and pharmacodynamics studies of nicotine after oral administration in mice: effects of methoxsalen, a CYP2A5/6 inhibitor. Nicotine Tob Res 16, 18-25.

Auerbach, O., Garfinkel, L., 1989. Histologic changes in the urinary bladder in relation to cigarette smoking and use of artificial sweeteners. Cancer 64, 983-987.

Baris, D., Karagas, M.R., Verrill, C., Johnson, A., Andrew, A.S., Marsit, C.J., Schwenn, M., Colt, J.S., Cherala, S., Samanic, C., Waddell, R., Cantor, K.P., Schned, A., Rothman, N., Lubin, J., Fraumeni, J.F., Jr., Hoover, R.N., Kelsey, K.T., Silverman, D.T., 2009. A case-control study of smoking and bladder cancer risk: emergent patterns over time. J Natl Cancer Inst 101, 1553-1561.

Bavarva, J.H., Tae, H., McIver, L., Garner, H.R., 2014. Nicotine and oxidative stress induced exomic variations are concordant and overrepresented in cancer-associated genes. Oncotarget 5, 4788-4798.

Beckel, J.M., Kanai, A., Lee, S.-J., de Groat, W.C., Birder, L.A., 2006. Expression of functional nicotinic acetylcholine receptors in rat urinary bladder epithelial cells. Am J Physiol Renal Physiol 290, F103-110.

Bedard, K., Krause, K.H., 2007. The NOX family of ROS-generating NADPH oxidases: physiology and pathophysiology. Physiol Rev 87, 245-313.

Cohen, S.M., Boobis, A.R., Meek, M.E., Preston, R.J., McGregor, D.B., 2006. 4Aminobiphenyl and DNA reactivity: case study within the context of the 2006 IPCS Human Relevance Framework for Analysis of a cancer mode of action for humans. Crit Rev Toxicol 36, 803-819.

Cohen, S.M., Fisher, M.J., Sakata, T., Cano, M., Schoenig, G.P., Chappel, C.I., Garland, E.M., 1990. Comparative analysis of the proliferative response of the rat urinary bladder to sodium saccharin by light and scanning electron microscopy and autoradiography. Scanning Microsc 4, 135-142.

Cohen, S.M., Ohnishi, T., Clark, N.M., He, J., Arnold, L.L., 2007. Investigations of rodent urinary bladder carcinogens: collection, processing, and evaluation of urine and bladders. Toxicol Pathol 35, 337-347.

Dasgupta, P., Rizwani, W., Pillai, S., Kinkade, R., Kovacs, M., Rastogi, S., Banerjee, S., Carless, M., Kim, E., Coppola, D., Haura, E., Chellappan, S., 2009. Nicotine induces cell proliferation, invasion and epithelial-mesenchymal transition in a variety of human cancer cell lines. Int J Cancer 124, 36-45.

Dodmane, P.R., Arnold, L.L., Pennington, K.L., Cohen, S.M., 2014. Orally administered nicotine induces urothelial hyperplasia in rats and mice. Toxicology 315 , 49-54.

Fuji, S., Suzuki, S., Naiki-Ito, A., Kato, H., Hayakawa, M., Yamashita, Y., Kuno, T., Takahashi, S., 2017. The NADPH oxidase inhibitor apocynin suppresses preneoplastic liver foci of rats. Toxicol Pathol 45, 544-550.

Hukkanen, J., Jacob, P., 3rd, Benowitz, N.L., 2005. Metabolism and disposition kinetics of nicotine. Pharmacol Rev 57, 79-115.

IARC, 2004. Tobacco smoking. WHO Press, Lyon, France, pp. 585. 
Jiang, J., Yuan, X., Zhao, H., Yan, X., Sun, X., Zheng, Q., 2014. Licochalcone A inhibiting proliferation of bladder cancer $\mathrm{T} 24$ cells by inducing reactive oxygen species production. Biomed Mater Eng 24, 1019-1025.

Kato, A., Naiki-Ito, A., Nakazawa, T., Hayashi, K., Naitoh, I., Miyabe, K., Shimizu, S., Kondo, H., Nishi, Y., Yoshida, M., Umemura, S., Hori, Y., Mori, T., Tsutsumi, M., Kuno, T., Suzuki, S., Kato, H., Ohara, H., Joh, T., Takahashi, S., 2015. Chemopreventive effect of resveratrol and apocynin on pancreatic carcinogenesis via modulation of nuclear phosphorylated GSK3beta and ERK1/2. Oncotarget 6, 4296342975.

Minna, J.D., 2003. Nicotine exposure and bronchial epithelial cell nicotinic acetylcholine receptor expression in the pathogenesis of lung cancer. J Clin Invest 111, 31-33.

Miyata, Y., Matsuo, T., Sagara, Y., Ohba, K., Ohyama, K., Sakai, H., 2017. A MiniReview of Reactive Oxygen Species in Urological Cancer: Correlation with NADPH Oxidases, Angiogenesis, and Apoptosis. Int J Mol Sci 18.

Ohnishi, T., Arnold, L.L., He, J., Clark, N.M., Kawasaki, S., Rennard, S.I., Boyer, C.W., Cohen, S.M., 2007. Inhalation of tobacco smoke induces increased proliferation of urinary bladder epithelium and endothelium in female C57BL/6 mice. Toxicology 241, 58-65.

Pervaiz, S., Cao, J., Chao, O.S., Chin, Y.Y., Clement, M.V., 2001. Activation of the RacGTPase inhibits apoptosis in human tumor cells. Oncogene 20, 6263-6268. Qiu, M., Chen, L., Tan, G., Ke, L., Zhang, S., Chen, H., Liu, J., 2015. A reactive oxygen species activation mechanism contributes to JS-K-induced apoptosis in human bladder cancer cells. Sci Rep 5, 15104.

Stefanska, J., Pawliczak, R., 2008. Apocynin: molecular aptitudes. Mediators Inflamm 2008, 106507.

Stolk, J., Hiltermann, T.J., Dijkman, J.H., Verhoeven, A.J., 1994. Characteristics of the inhibition of NADPH oxidase activation in neutrophils by apocynin, a methoxysubstituted catechol. Am J Respir Cell Mol Biol 11, 95-102.

Suzuki, S., Arnold, L.L., Pennington, K.L., Kakiuchi-Kiyota, S., Cohen, S.M., 2009. Effects of co-administration of dietary sodium arsenite and an NADPH oxidase inhibitor on the rat bladder epithelium. Toxicology 261, 41-46.

Suzuki, S., Cohen, S.M., Arnold, L.L., Kato, H., Fuji, S., Pennington, K.L., Nagayasu, Y., Naiki-Ito, A., Yamashita, Y., Takahashi, S., 2018. Orally administered nicotine effects on rat urinary bladder proliferation and carcinogenesis. Toxicology 398-399, 3140 .

Suzuki, S., Cohen, S.M., Arnold, L.L., Pennington, K.L., Kato, H., Naiki, T., Naiki-Ito, A., Yamashita, Y., Takahashi, S., 2020. Cotinine, a major nicotine metabolite, induces cell proliferation on urothelium in vitro and in vivo. Toxicology 429, 152325.

Suzuki, S., Pitchakarn, P., Sato, S., Shirai, T., Takahashi, S., 2013a. Apocynin, an NADPH oxidase inhibitor, suppresses progression of prostate cancer via Rac1 dephosphorylation. Exp Toxicol Pathol 65, 1034-1041.

Suzuki, S., Shiraga, K., Sato, S., Punfa, W., Naiki-Ito, A., Yamashita, Y., Shirai, T., Takahashi, S., 2013b. Apocynin, an NADPH oxidase inhibitor, suppresses rat prostate carcinogenesis. Cancer Sci 104, 1711-1717. 


\section{Figure legends}

Fig. 1. Histopathology of urinary bladder and renal pelvis. Simple hyperplasia in control rats (40 ppm nicotine) and normal urothelium in the urinary bladder of rats coadministered nicotine (40 ppm) and apocynin (250 or $750 \mathrm{mg} / \mathrm{kg})(\mathrm{A})$, simple hyperplasia in both control (40 ppm nicotine) and the renal pelvis of rats co-administered nicotine (40 ppm) and apocynin (250 or $750 \mathrm{mg} / \mathrm{kg})(\mathrm{B})$. Bars $=50 \mu \mathrm{m}$.

Fig. 2. WST-1 assay and ROS production with apocynin and/or cotinine on human bladder cancer cell lines. Cell viabilities with apocynin (A), and with apocynin and/or cotinine (B) on T24 and UMUC3 cells. ROS production data detected by DCFH-DA after apocynin treatment for $24 \mathrm{hrs}(\mathrm{C})$. The average of optical density or intensity in the control is set as 1.0.**,***: $\mathrm{P}<0.01,0.001$ compared to untreated control. \#\#, \#\#\#: $\mathrm{P}$ $<0.01$ and 0.001 compared to both apocynin and cotinine treated cells.

Supplementary Fig. 1. SEM examination of urinary bladder. Class 4 in the urinary bladder of control rats (40 ppm nicotine) and Class 3 in the urinary bladder of rats coadministered nicotine $(40 \mathrm{ppm})$ and apocynin $(250$ or $750 \mathrm{mg} / \mathrm{kg})$ Bars $=100 \mu \mathrm{m}$. 
Table 1 Body and organ weights, water and food consumptions

\begin{tabular}{|c|c|c|c|c|c|c|c|c|c|}
\hline \multirow{2}{*}{ Treatment } & \multirow{2}{*}{ No. of rat } & \multirow{2}{*}{$\begin{array}{l}\text { Body weight }^{\mathrm{a}} \\
\text { (g) }\end{array}$} & \multicolumn{2}{|c|}{ Liver $^{a}$} & \multicolumn{2}{|c|}{ Kidneys $^{\mathrm{a}}$} & \multirow{2}{*}{$\begin{array}{l}\text { Water consumption }^{a} \\
\text { (ml/rat/day) }\end{array}$} & \multirow{2}{*}{$\begin{array}{c}\text { Food consumption }{ }^{a} \\
\text { (g/rat/day) }\end{array}$} & \multirow{2}{*}{$\begin{array}{c}\text { Apocynin intake } \\
\text { (mg/rat/day) }\end{array}$} \\
\hline & & & Absolute (g) & Relative (\%) & Absolute (g) & Relative $(\%)$ & & & \\
\hline Control (40 ppm nicotine) & 13 & $212.2 \pm 9.0$ & $6.7 \pm 0.3$ & $3.2 \pm 0.1$ & $1.6 \pm 0.1$ & $7.5 \pm 0.3$ & $15.1 \pm 1.0$ & $13.2 \pm 2.1$ & - \\
\hline $40 \mathrm{ppm}$ nicotine $+250 \mathrm{mg} / \mathrm{kg}$ apocynin & 12 & $213.1 \pm$ & $7.0 \pm 0.4$ & $3.3 \pm 0.1$ & $1.7 \pm 0.1$ & $7.8 \pm 0.2$ & $15.5 \pm 1.2$ & $14.6 \pm 2.9$ & $3.6 \pm 0.7$ \\
\hline $40 \mathrm{ppm}$ nicotine $+750 \mathrm{mg} / \mathrm{kg}$ apocynin & 12 & $214.3 \pm$ & $7.0 \pm 0.5$ & $3.3 \pm 0.1$ & $1.7 \pm 0.1$ & $7.8 \pm 0.2$ & $15.5 \pm 1.2$ & $15.3 \pm 2.9$ & $11.5 \pm 2.2$ \\
\hline
\end{tabular}

a.$$
(\mathrm{g})
$$

Table 2 The effects of treatment with nicotine and apocynin for 4 weeks on the bladder urothelium

\begin{tabular}{|c|c|c|c|c|c|c|c|c|c|c|}
\hline \multirow{2}{*}{ Treatment } & \multirow{2}{*}{ No. of rat } & \multirow{2}{*}{$\begin{array}{c}\text { Simple } \\
\text { hyperplasia }\end{array}$} & \multirow{2}{*}{ Ki67 (\%) } & \multirow{2}{*}{ TUNEL (\%) } & \multirow{2}{*}{ 8-OHdG (\%) } & \multicolumn{5}{|c|}{ SEM Classification } \\
\hline & & & & & & 1 & 2 & 3 & 4 & 5 \\
\hline Control (40 ppm nicotine) & 13 & 13 & $1.8 \pm 0.8$ & $1.34 \pm 0.47$ & $0.84 \pm 0.36$ & 0 & 0 & 6 & 7 & 0 \\
\hline 40 ppm nicotine + Apocynin $750 \mathrm{mg} / \mathrm{kg}$ & 12 & $7^{*}$ & $0.7 \pm 0.6^{* *}$ & $0.69 \pm 0.29^{* * *}$ & $0.36 \pm 0.16^{* * *}$ & 0 & 0 & 4 & 8 & 0 \\
\hline
\end{tabular}

${ }^{*},{ }^{* *},{ }^{* * *}$ : Significantly different from control group, $\mathrm{P}<0.05,0.01,0.001$

Table 3 The effects of treatment with nicotine and apocynin for 4 weeks on the kidney pelvis urothelium

\begin{tabular}{lcccccc}
\hline \multicolumn{1}{|c}{ Treatment } & No. of rat & $\begin{array}{c}\text { Simple } \\
\text { hyperplasia }\end{array}$ & Ki67 (\%) & TUNEL (\%) & 8-OHdG (\%) \\
\hline Control (40 ppm nicotine) & 13 & 13 & $2.1 \pm 0.4$ & $0.13 \pm 0.12$ & $0.02 \pm 0.04$ \\
40 ppm nicotine + Apocynin 250mg/kg & 12 & 12 & $1.5 \pm 0.5^{* *}$ & $0.08 \pm 0.17$ & $0.02 \pm 0.04$ \\
40 ppm nicotine + Apocynin 750mg/kg & 12 & 12 & $1.5 \pm 0.3^{* *}$ & $0.08 \pm 0.10$ & $0.01 \pm 0.03$ \\
\hline$* *$ Signin
\end{tabular}

**: Significantly different from Control group, $\mathrm{P}<0.01$ 


\section{A. Urinary bladder}

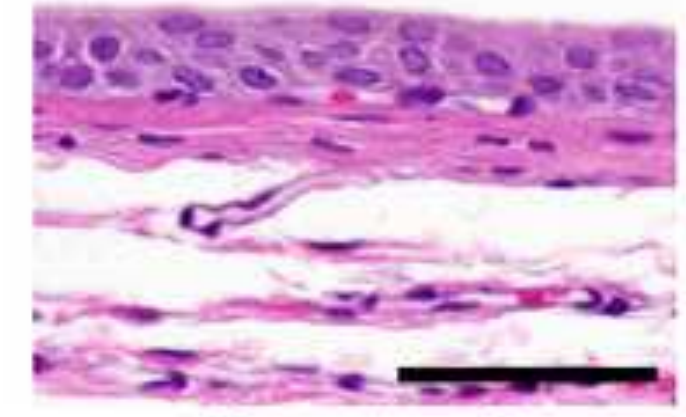

Control

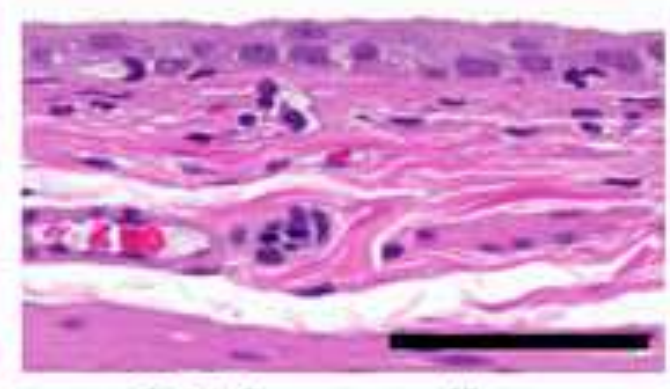

$250 \mathrm{mg} / \mathrm{kg}$

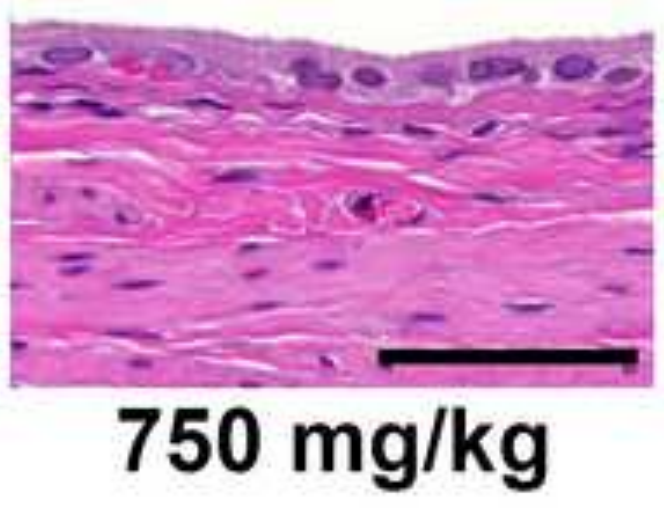

B. Renal pelvis

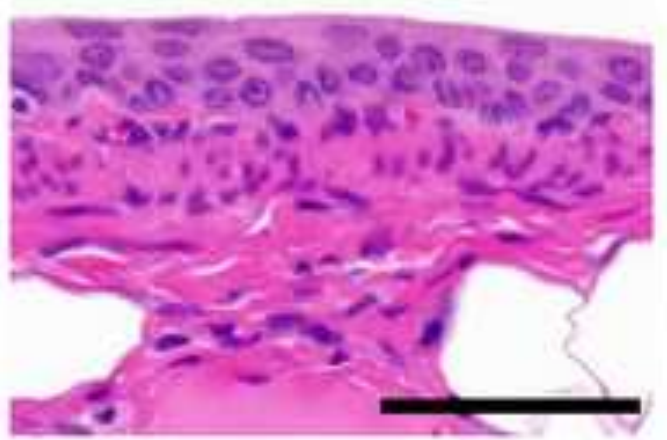

Control

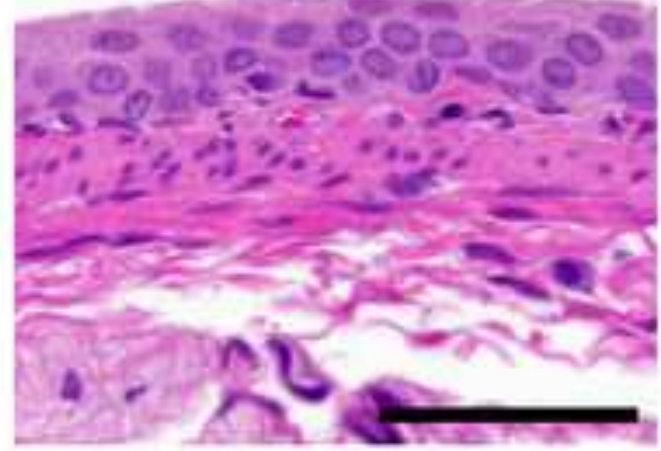
$250 \mathrm{mg} / \mathrm{kg}$

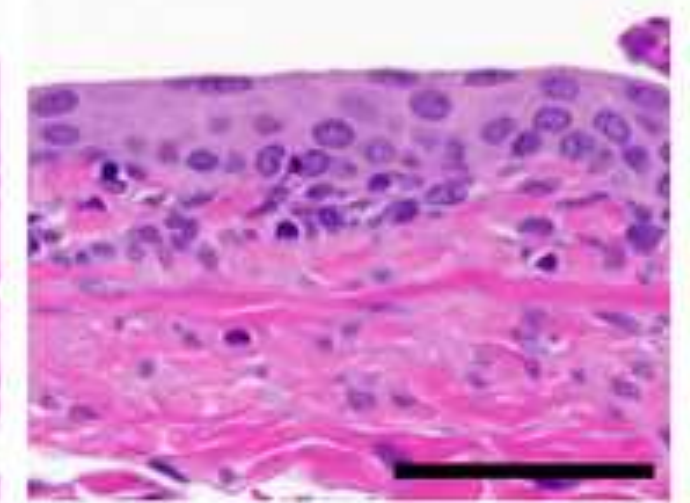

$750 \mathrm{mg} / \mathrm{kg}$ 
A
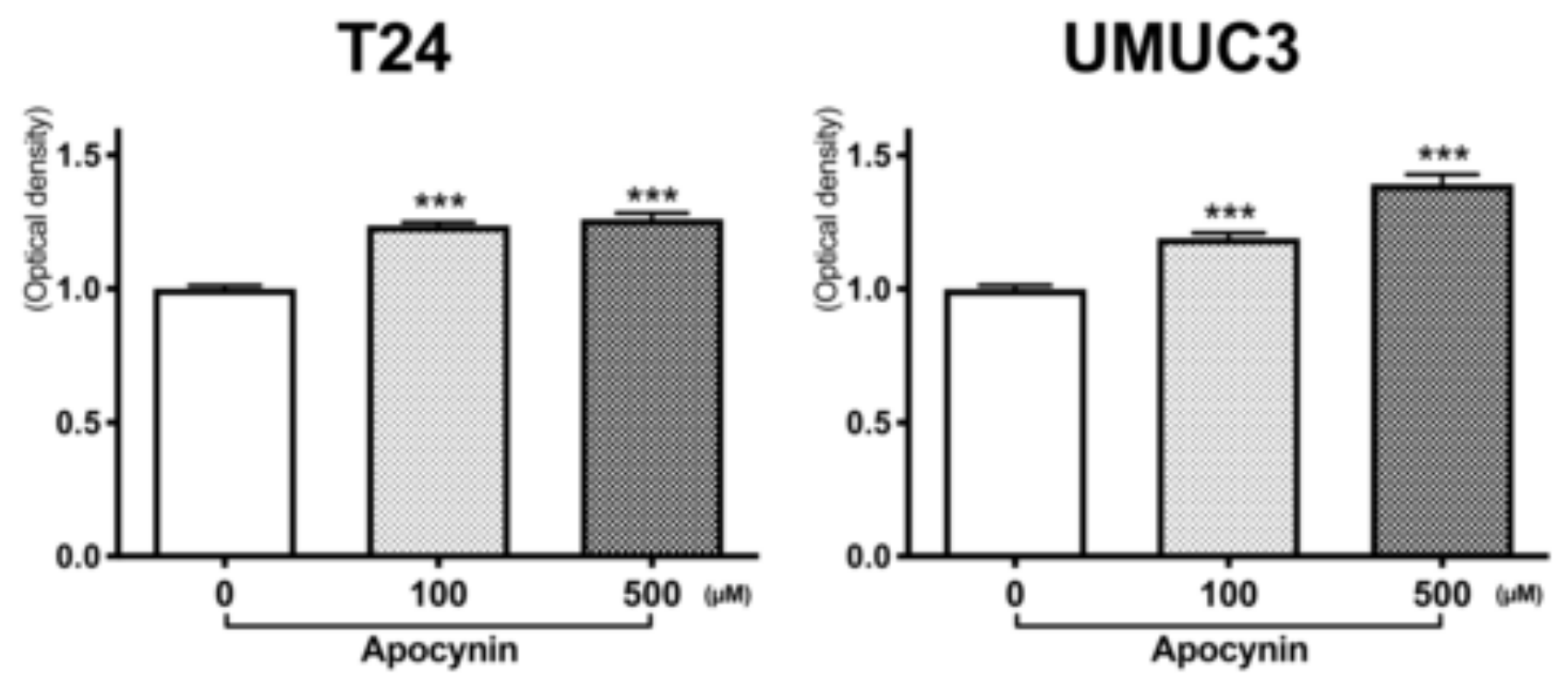

B
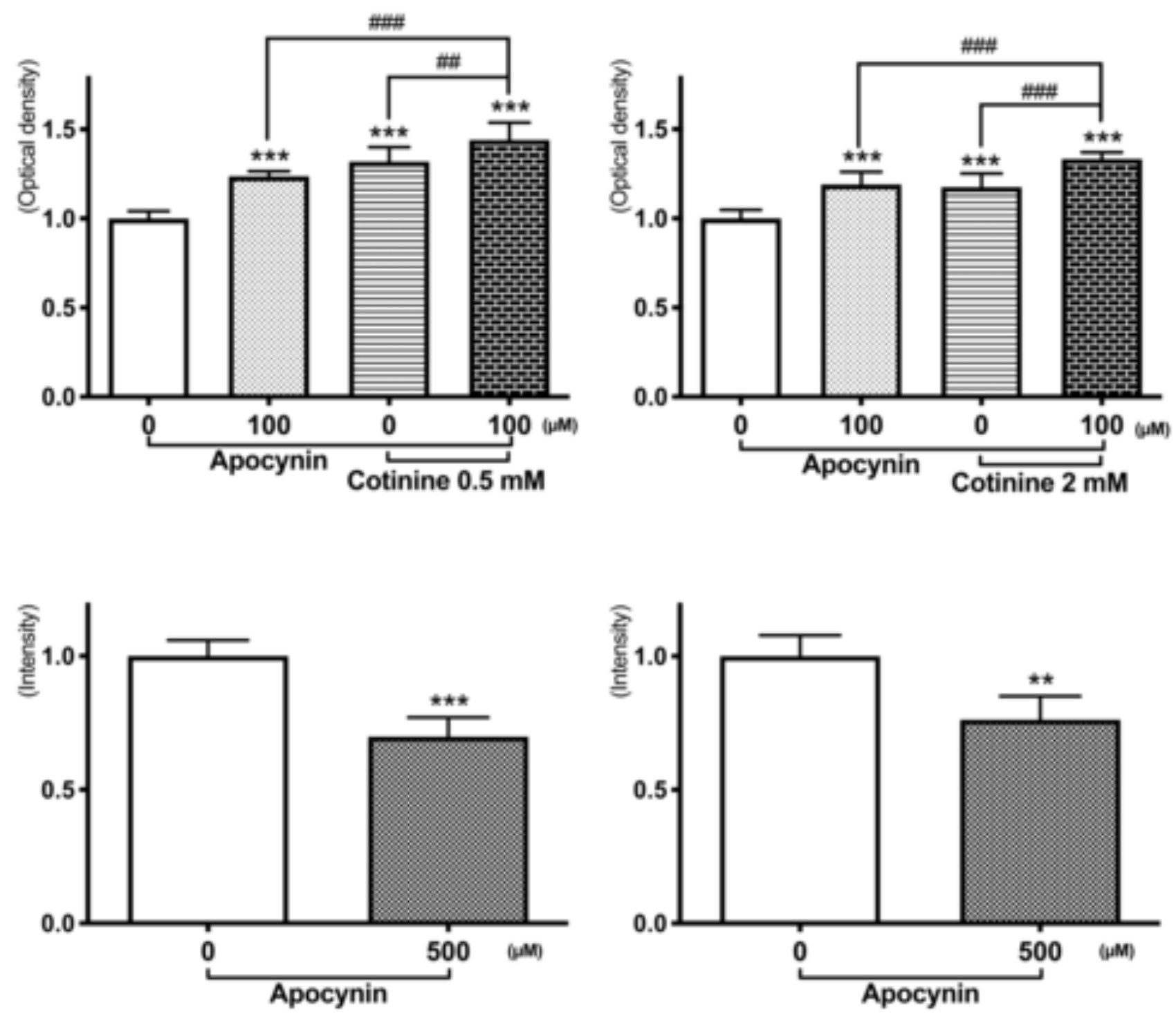